\title{
Random Scattering by Atomic Density Fluctuations in Optical Lattices
}

\author{
M. Blaauboer, ${ }^{\mathrm{a}, \mathrm{b}}$ G. Kurizki, ${ }^{\mathrm{a}}$ V.M. Akulin, ${ }^{\mathrm{c}}$ \\ ${ }^{a}$ Chemical Physics Department, Weizmann Institute of Science, Rehovot 76100, Israel \\ ${ }^{b}$ Department of Interdisciplinary Studies, Faculty of Engineering, Tel Aviv University, Tel Aviv 69978, Israel \\ ${ }^{c}$ Laboratoire Aimé Cotton, CNRS II, Bâtiment 505, Orsay Cedex 91405 France
}

(November 21, 2018)

We investigate hitherto unexplored regimes of probe scattering by atoms trapped in optical lattices: weak scattering by effectively random atomic density distributions and multiple scattering by arbitrary atomic distributions. Both regimes are predicted to exhibit a universal semicircular scattering lineshape for large density fluctuations, which depend on temperature and quantum statistics.

PACS numbers: 32.80.Pj, 05.40.-a, 42.50.Lc, 71.23.-k

Recent advances in trapping and manipulation of cold atoms interacting with external fields have primarily been implemented thus far either in single-atom systems, such as sparse (low-density) optical lattices [1], or in Bose-Einstein condensates with macroscopic numbers of atoms [2].

Between these two limiting regimes lies the scarcely investigated domain of processes involving a finite number of interacting atoms. Both the potential interest and the difficulties involved in studying

such processes are evident in the example of optical lattices with appreciable atomic filling factors [3], in

which the transition between "insulating" (localized) and "metallic" (superfluid) phases have been studied in the framework of the Bose-Hubbard model [4]. As confirmed by the above study, the mean-field approximation is inadequate for small numbers of interacting atoms in lattices due to the presence of large quantum fluctuations. Still more difficult is the analysis of fluctuations in systems of cold atoms coupled by long-range $(1 / r$ or $\left.1 / r^{3}\right)$ field-induced interactions [5]. This leads to the intruiging question: Is there a way to circumvent the formidable task of treating the full dynamics of such systems and still infer their important characteristics, e.g., their dependence on temperature, quantum statistics (Bose or Fermi), number of atoms and lattice parameters? And: Are there universal measurable features which can be a "signature" of the statistical ensemble (distribution function) of such systems?

Here we consider the possibility of inferring such statistical characteristics from the spectral features of probe photons or particles that are scattered by the density fluctuations of trapped atoms, notably in optical lattices, in two hitherto unexplored scenarios: (a) The probe is weakly (perturbatively) scattered by the local atomic density corresponding to the random occupancy of dif- ferent lattice sites (Fig. 1 - inset a). (b) The probe is multiply scattered by an arbitrary (possibly unknown a priori) multi-atom distribution in the lattice (Fig. 1 - inset b).

At the heart of our analysis is the idea that the Green function of the scattered photon or particle, which embodies the relevant spectral information, can be qualitatively estimated without resorting to cumbersome perturbative calculations of the probe-multiatom interaction by replacing this interaction Hamiltonian by an equivalent random matrix. The random matrix approach, which has been successfully applied to various disordered systems [6], allows the evaluation of probe spectra

to all orders of scattering, expressing them by means of only the first two moments (the mean and variance) of the random interaction, averaged over the statistical ensemble of the multiatom system. The highlight of our analysis, based on this random matrix approach, is the prediction of a semicircular spectral lineshape of the probe scattering in the large-fluctuation limit of trapped atomic ensembles. Thus far, the only known case of quasisemicircular lineshapes in optical scattering has been predicted [7] and experimentally verified 8 in dielectric microspheres with randomly distributed internal scatterers.

The Green function of the probe $(\mathrm{P})$ at energy $\epsilon=\hbar \omega$ is given by

$$
G_{P}(\epsilon)=\operatorname{Tr}_{S}\left[\frac{1}{\epsilon-\hat{H}_{P}-\hat{V}} \hat{\rho}_{S}\right]
$$

where $\hat{H}_{P}, \hat{V}$ and $\hat{\rho}_{S}$ are, respectively,

the unperturbed probe Hamiltonian, the probe-system interaction Hamiltonian and the density operator for the ensemble of the multiatom system (S). We shall assume that the following conditions hold. (i) There is no appreciable back-effect of the probe on the multiatom system (otherwise it is no longer a probe). (ii) The state of the multiatom system does not change during the interaction time with the probe, i.e., the multiatom system remains "frozen", as is applicable for optical or atomic probing. This situation then cannot be described as Markovian relaxation (exponential decay) of the probe state into the multiatom reservoir, since the correlation time of this reservoir is now much longer than that of the probe, in contrast with the basic assumption

of relaxation. (iii) The probe spectrum is broadband, i.e., it encompasses many of its eigenstates. 
For an ensemble "frozen" during the interaction time, the tracing in (11) implies statistical averaging over repeated realizations of the multiatom system, every time the probe scattering is recorded, or taking the expectation value with respect to the quantum state of the system. For simplicity, let us explicitly consider elastic scattering (the extension

to inelastic scattering is straightforward), for which

$$
\begin{array}{ll}
\hat{V}=\sum_{\vec{k}} f_{\vec{k}} \hat{\rho}_{P \vec{k}}^{\dagger} \hat{\rho}_{S \vec{k}}+\text { h.c. } & \text { or } \\
\hat{V} & =\sum_{\vec{k}} f_{\vec{k}} a_{\vec{k}}^{\dagger} \hat{\rho}_{S \vec{k}}+\text { h.c. }
\end{array}
$$

Here $f_{\vec{k}}$ is the scattering amplitude for momentum exchange $\hbar \vec{k}$ between the probe and the system and the $\vec{k}$-mode Fourier components of the probe (system) density operators $\hat{\rho}_{P \vec{k}}\left(\hat{\rho}_{S \vec{k}}\right)$ are defined in terms of their respective creation and annihilation operators $\hat{\rho}_{P \vec{k}}=$ $\sum_{\vec{q}} a_{\vec{q}}^{\dagger} a_{\vec{q}+\vec{k}}, \hat{\rho}_{S \vec{k}}=\sum_{\vec{q}} c_{\vec{q}}^{\dagger} c_{\vec{q}+\vec{k}}$. Equations (2a) and (2b) stand, respectively, for bilinear and linear probe-system coupling. For optical probes (2a) and (2b) correspond to Raman and single-photon scattering, respectively. For atom or neutron probes the coupling (2a) is appropriate.

The Green function (1) is obtainable, to all orders in $\hat{V}$ [9], by solving the set of equations for its diagonal elements

$$
G_{\vec{k} \vec{k}}(\epsilon)=\left[\epsilon-\epsilon_{\vec{k}}-\sum_{\vec{k}^{\prime}}\left\langle\hat{V}_{\vec{k} \vec{k}^{\prime}}^{2}\right\rangle G_{\vec{k}^{\prime} \vec{k}^{\prime}}(\epsilon)\right]^{-1},
$$

where $\epsilon_{\vec{k}}$ are the probe energy eigenvalues in the absence of potential fluctuations and pointed brackets denote the expectation value. The spectral information contained in these $G_{\vec{k} \vec{k}}$ is given by the density of states (DOS) of the probe $g(\epsilon)=-\frac{1}{\pi} \operatorname{Im} \sum_{\vec{k}} G_{\vec{k} \vec{k}}(\epsilon)$.

In order to extract information on the system we shall make two simplifying assumptions regarding the probe and the coupling potential (2): (i) $f_{\vec{k}}$ is flat in $\vec{k}$ (the coupling is strongly localized in space) within a band exceeding the relevant band of the system, so that $f_{\vec{k}} \approx f$; (ii) the statistical distribution of the probe is also flat in $\vec{k}$ and its second moment in $\left\langle\hat{V}^{2}\right\rangle$ is replacable by the square of its mean flux (or density) $\bar{n}_{P}^{2}$ for the bilinear coupling (2a) or by its mean flux (density) $\bar{n}_{P}$ for the linear coupling (2b). Under these assumptions we can rewrite the squared coupling potential in (3) as

$$
\left\langle\hat{V}_{\vec{k} \vec{k}^{\prime}}^{2}\right\rangle=\left\langle\hat{V}_{\vec{k} \vec{k}^{\prime}}\right\rangle^{2}+\mathcal{F}_{P} \mathcal{S}_{\vec{k} \vec{k}^{\prime}}
$$

Here $\langle\hat{V}\rangle$ is the mean coupling potential and $\mathcal{F}_{P} \sim$ $|f|^{2} \bar{n}_{P}^{2}$ or $\mathcal{F}_{P} \sim|f|^{2} \bar{n}_{P}$ in the case of (2a) and (2b), respectively. The quantity of interest for the system is the Fourier-transformed density-density correlation of the atomic system

$$
\mathcal{S}_{\vec{k} \vec{k}^{\prime}}=\left\langle\hat{\rho}_{S \vec{k}}^{\dagger} \hat{\rho}_{S \vec{k}^{\prime}}\right\rangle+\text { c.c. }
$$

Its diagonal element $\mathcal{S}_{\vec{k} \vec{k}}$ is the static structure factor $\mathcal{S}_{\vec{k}}$, which is the Fourier transform of the van-Hove correlation function $\left\langle\hat{\rho}_{S}^{\dagger}(\vec{r}, t=0) \hat{\rho}_{S}\left(\vec{r}^{\prime}, t^{\prime}=0\right)\right\rangle$ for the spatial density fluctuations of the "frozen" atomic ensemble.

The difficulty of having to evaluate or measure the matrix elements $\mathcal{S}_{\vec{k} \vec{k}^{\prime}}$ is avoided for a spatially random density distribution of the atomic system, due to random site occupancy (Fig. 1, inset a) and short-range interaction with the probe (e.g., a neutron or thermal atom). The elements $\mathcal{S}_{\vec{k} \vec{k}^{\prime}}$ in (4) and (5) can then be replaced by the average of the structure factor over all relevant $\vec{k}$ :

$$
\mathcal{S}_{\vec{k} \vec{k}^{\prime}} \rightarrow \overline{\mathcal{S}}=\int d \vec{k} \mathcal{S}_{\vec{k}} \sim\left\langle\hat{n}_{S}^{2}\right\rangle-\left\langle\hat{n}_{S}\right\rangle^{2},
$$

where the right-hand side of $\overline{\mathcal{S}}$ denotes the local atomic density or number variance averaged over the ensemble.

The implications of evaluating the probe DOS $g(\epsilon)$ using (3)-(6) will be examined for random fluctuations about a mean scattering potential $\left\langle V_{S}(x)\right\rangle$ (corresponding to the mean atomic density distribution) that is $1 \mathrm{D}$ periodic. The "unperturbed" probe dispersion associated with $\left\langle V_{S}(x)\right\rangle$ is $\epsilon_{\vec{k}}=-2 J \cos \left(k_{x} d\right)+A, J$ being the hopping frequency, $d$ the lattice period and $A$ the band energy offset. This gives rise to the following expression for the Green function (3)

$G(\epsilon)=\left[\epsilon-\epsilon_{\vec{k}}-\left\langle W^{2}\right\rangle \sum_{\vec{k}^{\prime}}\left(\epsilon-\epsilon_{\vec{k}^{\prime}}-\Lambda(\epsilon)+i \Delta(\epsilon)\right)^{-1}\right]^{-1}$

Here $\left\langle W^{2}\right\rangle \equiv \mathcal{F}_{P} \overline{\mathcal{S}}, \Lambda(\epsilon)=\left\langle W^{2}\right\rangle / \sqrt{(\epsilon-A)^{2}-4 J^{2}}$ for $|\epsilon-A|>2 J, \Delta(\epsilon)=\left\langle W^{2}\right\rangle / \sqrt{4 J^{2}-(\epsilon-A)^{2}}$ for $|\epsilon-A|<$ $2 J$ and both zero otherwise. Figure 11 shows how the probe DOS $g(\epsilon)$ changes from that of a periodic band structure corresponding to the mean potential $\left\langle V_{S}(x)\right\rangle$ to a semicircular shape as the amount of fluctuations measured by $\left\langle W^{2}\right\rangle$ increases.

In the multiple-scattering scenario,

which pertains to resonantly scattered atomic probes or to intracavity optical probes (Fig. 1, inset b), semicircular lineshapes are

obtained even when the $\mathcal{S}_{\vec{k} \vec{k}^{\prime}}$ cannot be claimed to belong to a random distribution (Fig. 2, inset).

In the case of strongly-interacting atoms within a lattice site or longe-range intersite density correlations [5] the distribution may be quite intricate, corresponding to sharp peaks of $\mathcal{S}_{\vec{k} \vec{k}^{\prime}}$. Nevertheless, the universal spectral trends of Fig. 11 can be shown

to hold in this scenario, provided $\left\langle\hat{V}^{2}\right\rangle^{1 / 2} g_{0}(\epsilon) \gg 1$, $g_{0}(\epsilon)$ denoting the "unperturbed" probe DOS. This condition allows us to estimate $G_{\vec{k} \vec{k}}$ in (3) to all orders in $\hat{V}$, upon replacing the state of the atomic system by a gaussian random ensemble [6,7]. The result is the following 
universal formula $[7$ for the renormalized probe energy $\tilde{\epsilon}$ at a given input energy $\epsilon$

$$
\epsilon=\tilde{\epsilon}+\left\langle W^{2}\right\rangle \operatorname{Tr}_{P}\left(\frac{1}{\tilde{\epsilon}-\hat{H}_{P}-i 0}\right) .
$$

The use of (8) leads to a semicircular lineshape similar to the one in Fig. 1, as if the potential were random.

In order to illustrate the role of temperature, quantum statistics and the mean lattice potential in producing the semicircular lineshape, we proceed to evaluate $\left\langle W^{2}\right\rangle$ for several simple models:

1. The isolated-site limit: The tightly-bound Bose or Fermi distributions in a lattice can be estimated by taking the potential of each site to be that of a harmonic well of depth $V_{0}$. The isolated-site approximation holds for atoms in the lowest vibrational band, when the coupling energy is much smaller than the excitation energy to the next band $\llbracket, \sqrt{\left\langle W^{2}\right\rangle} \ll \hbar \omega_{\nu}=\frac{2 \pi \hbar}{\lambda} \sqrt{\frac{2 V_{0}}{m}}, \lambda$ being the wavelength of the laser light.

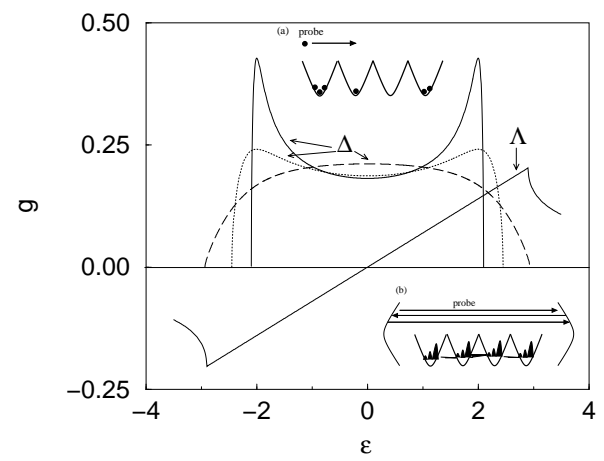

FIG. 1. Density of states $g(\epsilon)$ of a probe scattered by bosonic atoms in a 1D optical lattice. Solid, dotted and dashed curves stand for $\Delta(\epsilon)$ and the thick curve stands for $\Lambda(\epsilon)$ (dispersion), see text. All curves are numerically computed from $G(\epsilon)$ and correspond to average random couplings $\left\langle W^{2}\right\rangle=0.4,2$ and 10 respectively. The hopping frequency $J=1$, and for all curves $\int d \epsilon g(\epsilon)=1$. Inset a: A probe weakly scattered by a randomly occupied lattice. Inset b: A probe multiply scattered by a regular atomic distribution.

In the absence of additional external perturbations, the coupling $\left\langle W^{2}\right\rangle$ arises because of temperature-dependent fluctuations in the site-occupancy of the optical lattice, which has an approximately gaussian distribution [3]. The resulting random coupling energies averaged over all states yield $\left\langle W^{2}\right\rangle \sim \mathcal{F}_{P}\left\langle\left\langle n_{\vec{k}}, n_{\vec{k}^{\prime}}\right\rangle-\left\langle n_{\vec{k}}\right\rangle\left\langle n_{\vec{k}^{\prime}}\right\rangle\right\rangle_{\vec{k} \vec{k}^{\prime}}=$ $\mathcal{F}_{P}\left\langle\left\langle n_{i}, n_{j}\right\rangle-\left\langle n_{i}\right\rangle\left\langle n_{j}\right\rangle\right\rangle_{i j} \approx \mathcal{F}_{P}\left(\left\langle n_{S}^{2}\right\rangle-\left\langle n_{S}\right\rangle^{2}\right)$. Here $n_{\vec{k}} \equiv c_{\vec{k}}^{\dagger} c_{\vec{k}}$, $i$ and $j$ label atomic sites, and $\left\langle n_{S}\right\rangle$ is the average number of atoms per site. The last step applies whenever $n_{i} \approx n_{j} \forall i, j$ and the density fluctuations are approximately site-independent. We have verified this by numerical simulation, considering 2 to 4 identical atoms on a 1D lattice with 6 sites and calculating the density fluctuations if the probability of an occupied site is $1 / 10$ of the probability of an empty site. In all cases the maximum relative difference between $\mathcal{F}_{P} \mathcal{S}_{\vec{k} \vec{k}^{\prime}}$ and $\left\langle W^{2}\right\rangle$ was less than $10 \%$.

The kinetic contribution to $\left\langle W^{2}\right\rangle$ due to evaporation of atoms from the lattice is the dominant one at high temperatures, regardless of the statistics. If all the atoms are in the lowest energy band, we may adopt the rate equation used to describe the formation of electron-hole clusters in a plasma [10] and find

$$
\left\langle W^{2}\right\rangle_{\text {evap }}=a\left\langle n_{S}\right\rangle T^{2} e^{-\beta V_{0}} .
$$

Here $a=k_{B} m c_{p}$, with $k_{B}$ the Boltzmann constant, $m$ the mass of the atoms and $c_{p}$ their specific heat, $T$ denotes the temperature, $\beta^{-1} \equiv k_{B} T$ and $V_{0}$ is the optical lattice potential. The influence of evaporation becomes the dominant effect for $T \sim 25 \mu \mathrm{K}$. Around $T \sim 300 \mu \mathrm{K}$ these fluctuations become comparable in size to the square of the optical lattice potential $\left(\left\langle W^{2}\right\rangle \sim V_{0}^{2} \sim 100(\mathrm{neV})^{2}\right)$ and atoms then largely escape from the lattice.

At low temperatures (well below $100 \mu \mathrm{K}$ ) the densitydensity fluctuations depend on whether the atoms in the lattice are bosons or fermions. For bosonic atoms in the lowest vibrational state we obtain [11]

$$
\left\langle W^{2}\right\rangle_{\text {stat,Bose }}=\frac{z}{1-z}+\left(\frac{z}{1-z}\right)^{2}+\frac{d^{3}}{\lambda_{T}^{3}} \sum_{\alpha=1}^{\infty} \frac{z^{\alpha}}{\alpha^{1 / 2}} .
$$

Here we have approximated the motion of the atoms in the potential wells by a harmonic oscillation with frequency $\omega_{v}$ [12], $z \equiv e^{-k_{B} T / \hbar \omega_{v}}, d$ denotes the average lattice spacing and $\lambda_{T}=\left(2 \pi \hbar^{2} / m k_{B} T\right)^{1 / 2}$, the thermal wavelength, is the length scale separating quantum statistical behavior (for $\lambda_{T} \sim d$ ) from classical MaxwellBoltzmann behavior (for $\lambda_{T} \ll d$ ). For fermionic atoms in an optical lattice [13 one starts with the analog of the coupling (2) for particles

with spin, using creation and annihilation operators $c_{\vec{k} \sigma}^{\dagger}$ and $c_{\vec{k} \sigma}$ and performing an additional sum over the spin index $\sigma$, and follows the same analysis as above. One then finds

$$
\left\langle W^{2}\right\rangle_{\text {stat }, \text { Fermi }}=\frac{z}{1+z}+\left(\frac{z}{1+z}\right)^{2}+\frac{2 d^{3}}{\lambda_{T}^{2} \lambda_{F}} \sum_{\alpha=1}^{\infty} \frac{z^{\alpha}}{\alpha^{1 / 2}},
$$

with $\lambda_{F}$ the Fermi wavelength. At high temperatures $z \rightarrow 0$ and both (10) and (11) reduce to the classical Maxwell-Boltzmann result $\left\langle W^{2}\right\rangle_{\text {stat,clas }}=z$. At low temperatures, fermionic fluctuations approach a constant value, whereas bosonic fluctuations become very large as $T$ decreases below $\sim 1 \mu \mathrm{K}$, marking the Bose-Einstein condensation.

In Fig. 2 we have taken typical parameters for available optical lattices 12, 14, 3] to show how $\left\langle W^{2}\right\rangle$ evolves as a function of temperature for both bosonic and fermionic 
$\mathrm{Li}$ atoms. The total density-density fluctuations consist of the sum of (9) and either (10) or (11), depending on the statistics. The isolated-site condition is satisfed for the entire temperature range displayed in Fig. 2. Since the hopping frequency $J \sim V_{0}$, the random coupling for bosonic Li atoms changes from $\left\langle W^{2}\right\rangle / J^{2} \sim 10$ to $\left\langle W^{2}\right\rangle / J^{2} \sim 100$, when going from $T \sim 8 \mu \mathrm{K}$ to $T \sim 100 \mu \mathrm{K}$. Simultaneously the DOS then evolves from the periodic to the semicircular shape as in Fig. 1. The behavior for $\mathrm{Na}$ or $\mathrm{Rb}$ atoms is found to be similar to that of bosonic Li atoms, apart from their different mass values in (9) and (10). Employing currently achievable Bragg scattering techniques [14 with a far off-resonant laser $\left(1 \mathrm{~mW} / \mathrm{cm}^{2}\right.$ intensity, $5.2 \mathrm{MHz}$ detuning) and a lattice with a sufficiently high atomic filling factor [3], the scattering spectrum is expected to evolve with $\mathrm{T}$, in the microkelvin range, from the discrete to the semicircular regime.

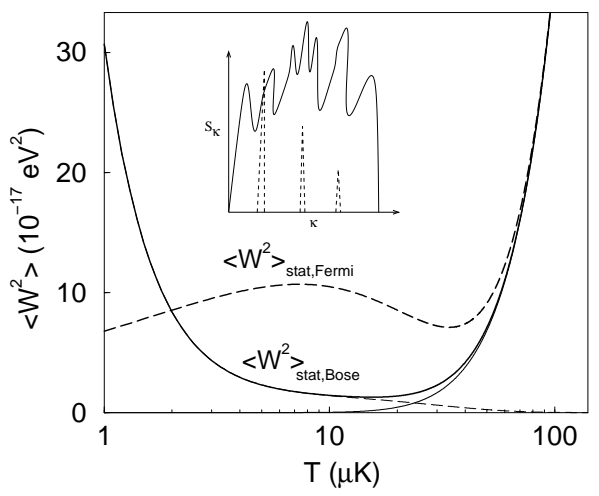

FIG. 2. Density-density fluctuations $\left\langle W^{2}\right\rangle$ (in units of 10 $\left.(\mathrm{neV})^{2}\right)$ as a function of temperature $\mathrm{T}$ for bosonic and fermionic Li atoms in an optical lattice. Thin solid line - fluctuations due to evaporation (9) (scaled by a factor of 7.8), thin dashed line - statistical fluctuations (10). Thick solid line - total fluctuations $\left\langle W^{2}\right\rangle$ for bosonic Li atoms; thick dashed line - their counterpart for fermionic Li atoms. Parameters used are: $V_{0}=5 \mathrm{neV},\left\langle n_{S}\right\rangle=0.1, \mathrm{~d}=0.1 \mu \mathrm{m}, c_{p}(\mathrm{Li}) \sim(3.6) \cdot 10^{6}$ $\mathrm{J} \mathrm{kg}^{-1} \mathrm{~K}^{-1}, \lambda_{F}(\mathrm{Li})=6 \cdot 10^{-10} \mathrm{~m}$ and $\omega_{v}(\mathrm{Li}) \sim 2 \cdot 10^{6} \mathrm{~s}^{-1}$ [12]. Inset: Static structure factor vs. $\kappa$ for phonons (solid curve) and nearly-free fermions (dashed curve) in a lattice at finite $\mathrm{T}$.

2. The nearly-free limit: A Bose or Fermi gas weakly modulated by the lattice potential yields $\mathcal{S}_{\vec{k} \vec{k}^{\prime}}=$ $\mathcal{S}_{\vec{\kappa}=\vec{k}-\vec{k}^{\prime}}=\left|\phi_{\vec{\kappa}}\right|^{2} \mathcal{S}_{\vec{\kappa}}^{\text {(free) }}$. Here $\vec{\kappa}$ is a reciprocal lattice vector, $\phi_{\vec{\kappa}}$ is the corresponding Fourier harmonic of the lattice potential (normalized to 1 ) and $\mathcal{S}_{\vec{\kappa}}^{(\text {free) }}$ is the structure factor for momentum transfer $\hbar \kappa$ in a free Bose or Fermi gas. For a Fermi gas $\mathcal{S}_{\vec{\kappa}}^{(\text {free })}= \pm \Theta\left(k_{f}-\kappa\right)$, the Fourier transform of pair correlations with parallel or anti-parallel spins (which determines the sign): it is the well-known step function which vanishes for $\kappa$ larger than the Fermi wavevector $k_{f}$. At finite temperatures this distribution broadens. The replacement of the nearly-free fermionic $\mathcal{S}_{\vec{k} \vec{k}^{\prime}}$ by the average value (6) is then justifiable only in the multiple-scattering scenario, while in the weak-scattering scenario the lattice potential harmonics $\phi_{\vec{k}}$ pick out well-defined $\mathcal{S}_{\vec{k}-\vec{k}^{\prime}=\vec{k}}$ (Fig. 2, inset - dashed line).

3. The phonon regime: Excitations at frequencies below the chemical potential of a Bose condensate trapped in a lattice can produce collective phonon modes 15] whose "frozen" spectrum is characterized by $\mathcal{S}_{\vec{\kappa}}=\sum_{\vec{q}}\left[\left(\left\langle n_{\vec{q}}\right\rangle+\right.\right.$ 1) $\left.\sum_{\vec{G}} \delta(\vec{\kappa}-\vec{q}-\vec{G})+\left\langle n_{\vec{q}}\right\rangle \sum_{\vec{G}} \delta(\vec{\kappa}+\vec{q}+\vec{G})\right]$, where $\left\langle n_{\vec{q}}\right\rangle$ is the mean number of phonons at temperature $T$ with wavevector $\vec{q}$, and $\vec{G}$ denotes the reciprocal lattice vector. The phonon mode spectrum includes quasi-local modes in the case of fluctuating atomic distributions. This naturally leads to the limit (6) and an effectively random coupling (Fig. 2, inset - solid line).

To conclude, we have identified novel regimes of probe scattering by atoms trapped in optical lattices in the random-density and multiple-scattering regimes. These regimes cannot be treated by the mean-field approximation, but are characterized by a universal feature of large density fluctuations, namely, semicircular scattering lineshapes. This is the atom-optical analog of the semicircular broadening of the DOS in disordered electronic systems, which as far as we know has not yet been observed unambiguously. The observation of this atom-optical counterpart presents a nontrivial but feasible challenge for experimentalists.

This work was supported by US-Israel BSF , the Israel Council for Higher Education, Minerva and Arc-en-Ciel.

[1] P. Verkerk et al., Phys. Rev. Lett. 68, 3861 (1992); M. Raizen et al., Phys. Today July 1997, 30 (1997); M. BenDahan et al., Phys. Rev. Lett. 76, 4508 (1996).

[2] A.S. Parkins and D.F. Walls, Phys. Rep. 303, 1 (1998); S. Inouye et al., Nature 392, 151 (1998); D.M. StamperKurn et al., Phys. Rev. Lett. 80, 2027 (1998); B.D. Anderson and M.A. Kasevich, Science 282, 1686 (1998); M.O. Scully, Phys. Rev. Lett. 82, 3927 (1999).

[3] M.T. DePue et al., Phys. Rev. Lett. 82, 2262 (1999).

[4] D. Jaksch et al., Phys. Rev. Lett. 81, 3108 (1998).

[5] D. O'Dell, S. Giovanazzi, G. Kurizki and V.M. Akulin, Phys. Rev. Lett. 84, 5687 (2000).

[6] M.L. Mehta, Random Matrices, (Academic, New York, 1991); B. Velicky et al., Phys. Rev. 179, 747 (1968).

[7] V.M. Akulin and G. Kurizki, Phys. Lett. A 174, 267 (1993); V.M. Akulin, Phys. Rev. A 48, 3532 (1993).

[8] D. Ngo and R.G. Pinnick, J. Opt. Soc. Am. A 11, 1352 (1994).

[9] P.W. Anderson, Phys. Rev. 124, 41 (1961). 
[10] C. Klingshirn and H. Haug, Phys. Rep. 70, 315 (1981).

[11] L.E. Reichl, A modern Course in Statistical Physics, (Wiley, New York, 1998).

[12] A. Kastberg et al., Phys. Rev. Lett. 74, 1542 (1995).

[13] M.R. Andrews, Nature 398, 195 (1999).

[14] G. Birkl et al., Phys. Rev. Lett. 75, 2823 (1995).

[15] D.M. Stamper-Kurn et al., Phys. Rev. Lett. 83, 2876 (1999). 